\title{
Osteochondroma as a Cause of Scapular Winging In an Adolescent Girl- Case Report.
}

\author{
Dr. S. P. Ilangovan, Dr. N. S. Rajarajan \\ Esic Hospital, Pgimsr, k.k. nagar, chennai.600078.INDIA.
}

\begin{abstract}
:
Introduction: Although long thoracic nerve palsy is the classical pathogenesis of winging scapula, it may also be caused by osteochondroma. We report a rare case of a scapular osteochondroma arising from the medial border and causing a pseudowinging of the scapula.

Case presentation:

15 year old girl presented with progressive deformity and painless swelling in the medial aspect of scapula. Clinical examination showed bony hard swelling in the medial aspect of scapula and had full painless range of movement with grating sensation on extreme range of movement. Patient was investigated and underwent open surgical excision. A histopathology examination confirmed the clinical diagnosis of osteochondroma. A clinical examination three months later showed a painless full range of motion and absence of grating sensation and disappearance of deformity.
\end{abstract}

Conclusion: osteochondroma should be considered in the differential diagnosis for any adolescent presents with a winging of the scapula.

Keywords: adolescent, benign tumor, osteochondroma, scapular winging.

\section{Introduction}

Osteochondroma is the most common primary benign tumor of the scapula with incidence of 3- 4.6 percent.(6). Usually it is a single lesion located on the anterior aspect of the scapula. It can cause winging of the scapula.

Winging of the scapula (scapula alata) is defined as the prominence of the medial border of scapula. In addition to pain and deformity, it can cause a reduction in the shoulder strength, and range of motion. This entity was first described by velpeau.(1). Serratus anterior muscle impairment is the classic etiopathology, secondary to long thoracic nerve palsy.(2).However, because a broad variety of different lesions may also account for winging of the scapula (3). Fiddian and King( 4) proposed a classification of scapula alata on an anatomic basis. Type 1 lesions are caused by nerve pathology, Type 2 lesion related to muscle pathology, Type 3 lesions relate to osseous pathology, Type 4 lesions include joint diseases.

The winged scapula can be either dynamic or static. In dynamic scapular winging, the omoplate becomes prominent on movement but demonstrates no pathological finding at rest. Scapula alata caused by serratus anterior palsy with insufficiency of retaining the omoplate to the thorax is the protype of dynamic winging.(2).The patient present with a decreased range of active shoulder motion, Shoulder weakness or shoulder discomfort on exertion. Onexamination, the scapula is abnormally high, moved, and winging occurs when the patient performs 90 degree forward flexion. Yet, dynamic winging can $\mathrm{b}$ caused by paralysis of the trapezius muscle. In this condition, winging occurs when the patient abducts the shoulder to 90 degree. By contrast, static winging of the scapula refer to cases where the scapula is prominent at rest and no appreciable change occurs with active movement of the shoulder. Therefore, some authors tend to describe this entity as pseudowinging.(3,5).

Osteochondromas are usually painless, but symptoms may result from complications such as mechanical restriction, fracture of bony stalk of the tumor, nerve impingement syndromes, malignant transformation of the cartilaginous cap and large bursa formation.

\section{Case Presentation;}

A 15 years old girl presented with a deformity and painless swelling in the right scapula and progressively increases for the past 6 months .She did not complain about any other symptoms. There was no history of trauma and family history was not contributory. A clinical examination showed an important asymmetry of her scapula with her right side elevated from her left side. A hard bony swelling was palpable along the medial border of right scapula. She had a complete painless and full ROM for both shoulder, but a grating sensation was felt on the right side when arm was passively abducted and elevated. Her neurological examination was normal. 
Plain radiographic evaluation in AP, lateral, oblique views were taken and showed a bony lesion in the medial border of the right scapula. The preoperative diagnosis was osteochondroma of the scapula and it was further evaluated by CT scan. Considering that the lesion was painless but it resulted in an important deformity, surgical resection was planned and performed under general anesthesia. The incision was performed along the medial border of the scapula. The osteochondroma was exposed and totally excised from the healthy scapular base removing the entire cartilaginous cap. (fig.1,2,3,4).A histopathology examination confirmed the clinical diagnosis. The patient was discharged on third post operative day. A clinical examination three months later showed full painless ROM and complete disappearance of the deformity.

\section{ILLUSTRATIONS;}

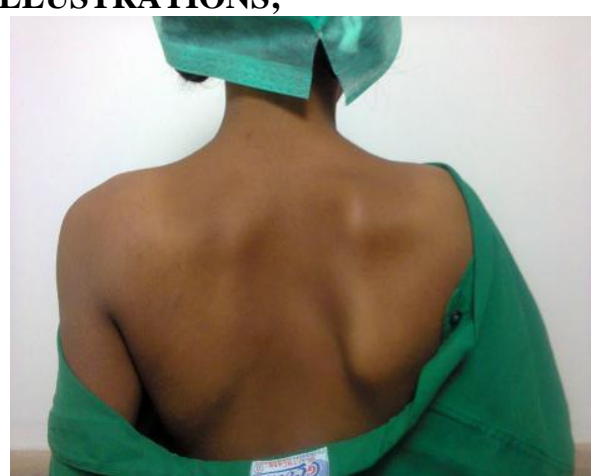

FIG-1

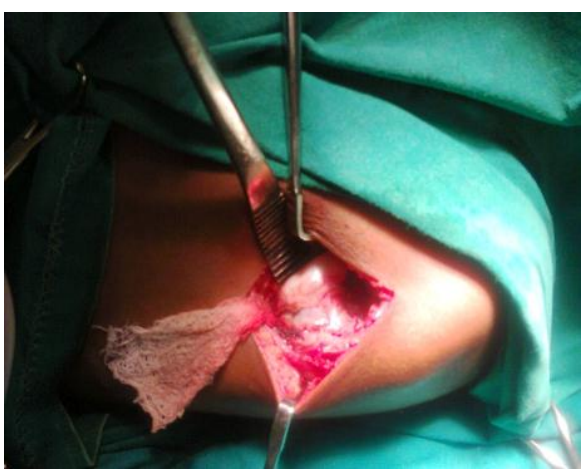

FIG-3

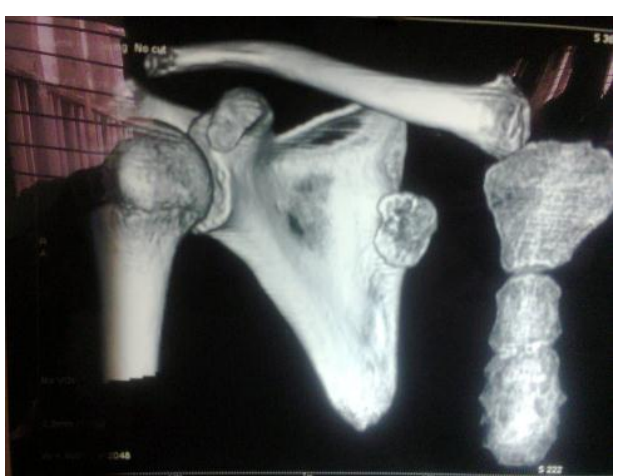

Fig-2

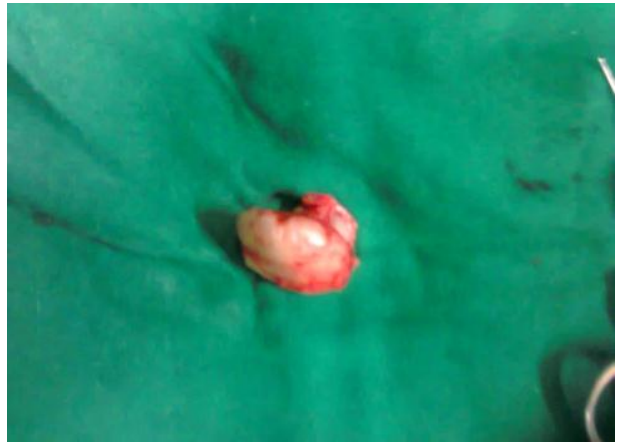

FIG -4

III. Disscussion

Though winging of scapula is generally considered to be synonymous with serratus anterior palsy. $(3,5)$. The differential diagnosis of winged scapula can be due to many causes other than serratus anterior palsy. A number of other causes have been described as pseudowinging. $(4,5)$.

The differential diagnosis of winged scapula can be complicated. When treating patients with scapular winging, orthopedic surgeon should be aware of different etiopathology. Although scapula alata usually indolent, patients with subscapular osteochondroma often complain of pain and grating sensation. On examination, an exostosis may be localized by internally rotating shoulder joint to produce grating or snapping and accentuated if a large accompanying bursa is formed. Audible crepitus may be produced with active abduction and internal rotation of the shoulder.(8).

As anteroposterior radiograpy may not always delineate the subscapular osteochondroma, a true lateral $\mathrm{x}$ - ray or a CT scan is necessary to demonstrate the mass and confirm the diagnosis. The pathognomonic radiographic picture is the the cortex of the host bone flaring into the cortex of exostosis and the the cancellous bone blending with each other. CT scan is not only useful for confirming diagnosis but also for preoperative planning.

MRI is recommended if malignancy is suspected. The rapid formation of bursa might be misinterpreted as malignant transformation of the cartilaginous cap of the osteochondroma.(5,7). MR images allow for a measurement of the thickness of the cartilaginous cap, which is a significant predictor of malignant transformation. A cap thinner than $1 \mathrm{~cm}$ usually indicates a benign condition, whereas a cap thicker than $2 \mathrm{~cm}$ generally corresponds to malignant transformation. The differential diagnosis between bursa and malignant transformation is important in patients with multiple osteochondroma. 
Malignant transformation to chondrosarcoma occurs in approximately $1 \%$ of solitary osteochondroma in adult life. Of interest, in patients with multiple hereditary exostosis, up to $27 \%$ may develop malignant transformation.(10). Finally MRI visualizes the effects of the lesions on surrounding structures.(5).A bone scintigraphy is weakly positive or negative in inactive exostoses of the adult but positive during malignant transformation and may be taken into consideration to exclude malignancy.(9). As biopsies carry the risk

of spreading the tumor content, this option is recommended only if imaging techniques are insufficient to assess the malignancy of the mass.

Operative treatment is recommended in the case of pain, decreased range of motion of the shoulder or local compression of nervous or vascular structures. Resection of osteochondroma is the treatment of choice for winged scapula caused by osteochondroma. If malignancy is suspected, operative resection is inevitable. Only the cartilaginous component is capable of growth and should be completely removed with its capsule. The prognosis of the solitary osteochondroma is excellent. Local recurrence after surgery is very rare. (9).

\section{Conclusion}

Dispite of its rarity osteochondroma should be considered in the differential diagnosis for any adolescent presenting with a winging of scapula.

\section{References}

[1] Bloch AM,Nevo Y,Ben-Sira L,Harel S,Shahar E. Winging of the scapula in a child with hereditary exostoses. Pediatr. Neurol.2002; 26; 74-76.

[2] Danielsson LG, el -Haddad I. Winged scapula due to osteochondroma. report of three children. Acta Orthop Scand.1989; 60; 728729

[3] Fiddian NJ, King RJ. The winged scapula. Clin Orthop.1984; 185; 228-236.

[4] Cooley LH, Torg JS. Pseudowinging of the scapula secondary to subscapular osteochondroma. Clin Orthop.1982; 162; $119-124$.

[5] Lynch AF,Fogarty EE,Dowling FE.Pseudowinging of the scapula due to osteochondroma. J. pediatr Orthop , 1985;5;722-724.

[6] Kumar N ,Ramachandran V ,Johnson GV,Southern S. Endoscopically assisted excision of subscapular osteochondroma. Acta Orthop Scand.1999; 70; 394-396.

[7] Milch H. Partial scapulectomy for snapping of the scapula. J Bone JOINT surgery Am.1950; 32-A; 561-566

[8] Parson TA. The snapping scapula and subscapular exostoses. J Bone and joint surgery Br.1973; 55; 345-349.

[9] Strizak AM, Cowen MH,. The snapping scapular syndrome. A case report. J Bone And Joint Surgery Am.1982;64;941-942.

[10] Garrison RC, Unni KK,,McLeod RA. Chondrosarcoma arising in osteochondroma. J Cancer; 1982, 49;1890-1897. 